\title{
Senescence regulates macrophage activation and angiogenic fate at sites of tissue injury in mice
}

\author{
Jennifer Kelly, Aslam Ali Khan, Jiyi Yin, Thomas A. Ferguson, and Rajendra S. Apte
}

Department of Ophthalmology and Visual Sciences, Washington University School of Medicine, St. Louis, Missouri, USA.

\begin{abstract}
Abnormal angiogenesis plays a key role in diseases of aging such as heart disease, certain cancers, and eye diseases including age-related macular degeneration. Macrophages have been shown previously to be both anti- and proangiogenic, and their role in regulating angiogenesis at sites of tissue injury is critical and complex. In this study, we analyzed cytokine gene expression patterns of mouse macrophages by realtime quantitative PCR and tested the functional effects of senescence on gene expression and macrophage polarization. Following laser injury to the retina, IL-10 was upregulated and Fas ligand (FasL), IL-12, and TNF- $\alpha$ were downregulated in ocular macrophages of old mice (>18 months of age). Downregulation of FasL on macrophages led to a loss of the antiangiogenic phenotype, as evidenced by the inability of these macrophages to inhibit vascular endothelial cells. Our results demonstrate that senescence, FasL, and IL-10 are key determinants of macrophage function that alter the growth of abnormal postdevelopmental blood vessels in disease processes including blinding eye disease.
\end{abstract}

\section{Introduction}

Immune vascular interactions can play an important role in regulating angiogenesis during development as well as in diseases of aging such as cancers, atheromatous heart disease, and blinding eye disease (1-4). There are several lines of evidence that suggest that macrophages play a critical role in this process. Macrophages have been established as necessary and sufficient in inducing regression of lens vasculature during embryonic development (5). They play a key role in inhibiting the growth of abnormal blood vessels in the eye in age-related macular degeneration (AMD), the leading cause of blindness in people over 50 years of age $(1,6)$. Blindness in AMD occurs largely from the exudative (wet) form of the disease, which is characterized by the development of abnormal blood vessels underneath the retina, i.e., choroidal neovascularization $(\mathrm{CNV})(7,8)$.

On the other hand, alternative lines of evidence have also implicated macrophages in promoting abnormal blood vessel growth in murine tissue injury models (9). In the tumor micromilieu, there is evidence that macrophage influence on angiogenesis is complex (10). These cells can be pro- or antiangiogenic depending on their polarization, which in turn is regulated by several cytokines in the tissue microenvironment (11-13). Recent studies have also demonstrated that $I L-10^{-/-}$mice are significantly impaired in their ability to generate $\mathrm{CNV}$ after laser-induced tissue injury to the eye (1). IL-10 promotes angiogenesis in the eye and inhibits antiangiogenic macrophage function at the site of tissue injury. This contrasts with the proangiogenic macrophage function that has been described in other CNV studies (9). Polarization of macrophages can play a pivotal role in determining the ultimate effector function of these cells (13). Macrophages stimulated in presence

Nonstandard abbreviations used: AMD, age-related macular degeneration; CNV, choroidal neovascularization; Ct, threshold cycle; FasL, Fas ligand; HMVEC, human dermal microvascular endothelial cell.

Conflict of interest: The authors have declared that no conflict of interest exists. Citation for this article: J. Clin. Invest. 117:3421-3426 (2007). doi:10.1172/JCI32430. of IFN- $\gamma$, LPS, or GM-CSF produce high levels of cytokines such as IL-12, IL-23, IL-6, and TNF- $\alpha$ with low levels of IL-10. This signature highlights an antiangiogenic macrophage that is also important in antibacterial and inflammatory functions. Our laboratory has previously demonstrated that GM-CSF-cultured macrophages can inhibit CNV upon injection in to the eyes of host mice at the time of tissue injury (1). In the presence of IL-10, IL-4, or IL-13, macrophages become polarized to a proangiogenic phenotype characterized by high levels of IL-10 and low levels of proinflammatory cytokines such as IL- 6 and TNF- $\alpha$.

It is important to understand the factors that regulate macrophage activity and determine their control of postdevelopmental angiogenesis, especially because abnormal angiogenesis in the aged eye in AMD leads to severe vision loss and blindness. In this study, we evaluated the effect of senescence on macrophage function and angiogenesis and its implications for aging-associated diseases such as AMD in a laser-induced model of angiogenesis. Laser-induced injury is a surrogate tissue injury model for CNV and has been an excellent predictor of therapies that have subsequently been approved for the treatment of this condition. We also explored the critical role of IL-10 in these processes.

\section{Results}

Aging is associated with enhanced angiogenesis. We have previously demonstrated that $I L-10^{-/-}$mice have significantly reduced ocular angiogenesis following laser injury (1). IL-10 is a critical regulator of macrophage migration and influx into the site of tissue injury and of the subsequent development of CNV (1). Regulation of ocular angiogenesis is particularly important in the elderly who develop CNV in the eye as a function of unbridled angiogenesis that eventually leads to blindness. In order to directly test the effect of tissue injury on angiogenesis in aged animals, we lasered the eyes of old mice (>18 months of age) and compared the CNV response on day 7 after laser injury with that of young mice ( $<3$ months of age). Old mice had significantly more $\mathrm{CNV}$ than did young mice (Figure 1), a finding similar to previous reports that tested mice 


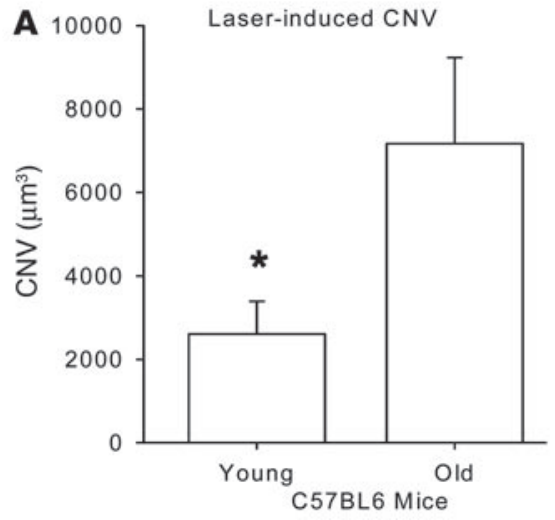

B

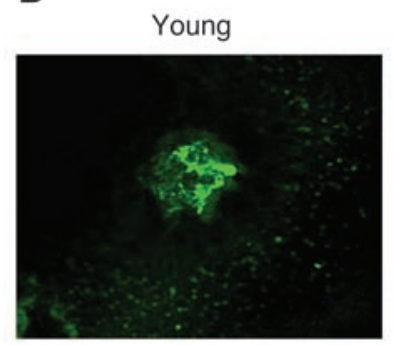

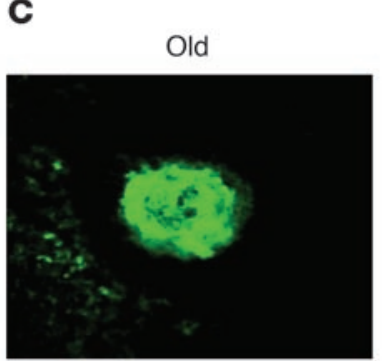

\section{Figure 1}

Quantification of CNV in young ( $<3$ months of age) and old ( $>18$ months of age) mice on day 7 following laser injury. Mice were perfused intracardially with FITC-dextran (green) prior to harvesting eyeballs and sclerochoroidal flat mounts were made in order to quantify CNV volumes by confocal microscopy. (A) Old mice demonstrated significantly more CNV than did young mice. Error bars represent SEM. ${ }^{\star} P<0.05$ vs. old. (B and C) Representative CNV from a young (B) and an old (C) mouse.

over 16 months of age (14). These findings suggest that antiangiogenic responses in old mice are impaired.

Macrophages from senescent mice fail to inhibit postdevelopmental angiogenesis. Because macrophages have been shown to inhibit angiogenesis after laser-induced injury to the eye, we isolated $\mathrm{F} 4 / 80^{+}$splenic macrophages from $\mathrm{C} 57 \mathrm{BL} / 6$ young or old mice by positive selection. In order to determine whether there is a functional difference between these macrophages in their ability to regulate angiogenesis, $1 \times 10^{5}$ macrophages from young and old mice were injected in to the eyes of old C57BL/ 6 host mice on the day of laser, and CNV response was measured on day 7 following laser injury. Macrophages from young mice significantly inhibited the CNV response, but macrophages from old mice failed to demonstrate any inhibition compared with the sham control mice injected with PBS (Figure 2). We have previously demonstrated that bone marrow-derived macrophages infiltrate into the laser lesions in the choroid after tissue injury (1). Here we isolated and dissected laser lesions as described in Methods, and F4/80 immunostaining demonstrated no significant differences in macrophage numbers within the laser lesions of young and old mice (data not shown). Enriched macrophage cytokine gene expression signature within these lesions was assessed by quantitative real-time PCR. Uninjured choroidal tissue was used as a control. We analyzed the cytokine profile of ocular macrophages isolated from young and old mice by measuring their cytokine gene expression 6 hours after tissue injury by real-time quantitative PCR. Macrophage-rich CNV lesions from old mice demonstrated significant downregulation of $I L-12$, TNF- $\alpha$, and Fas ligand (FasL) 6 hours after the laser-induced injury, while these genes were highly upregulated or relatively unaltered in young

\section{Figure 2}

Assessment of CNV inhibition following intraocular injection of macrophages $\left(1 \times 10^{5}\right.$ cells/eye). Old mice were injected with PBS (sham) or macrophages from either old or young mice on the day of laser, and CNV was measured at day 7 following laser injury. Macrophages isolated from young mice demonstrated significant inhibition of CNV following intraocular injection in to old eyes, while macrophages isolated from old mice fail to inhibit CNV. ${ }^{\star} P<0.02$, young vs. old. mice (Figure 3, A-C). In addition, $I L-6$ and $I L-23$ were upregulated after tissue injury in young mice at levels exponentially higher than those seen in old mice (Figure 3, D and E). IL-10 expression was upregulated in both young and old mice (Figure 3F).

$I L-10$ regulates macrophage function and promotes angiogenesis. These results are especially interesting given our previously published observations that $I L-10^{-/-}$mice, which lack native IL-10, have reduced angiogenesis after laser injury to the eye (1). IL-10 is a key regulator of macrophage polarization and regulation of angiogenesis $(1,13)$. We tested the ability of $I L-10^{-/-}$macrophages to inhibit CNV after intraocular injection to determine whether macrophage polarization affects angiogenic fate, i.e., CNV. Host C57BL/ 6 mice were injected intraocularly with $1 \times 10^{5} \mathrm{~F} 4 / 80^{+}$ splenic macrophages from either syngeneic $I L-10^{-/-}$mice or WT mice on the day of laser. Control mice were injected with PBS. Macrophages from $I L-10^{-/-}$mice inhibited CNV even more efficiently than did those from young syngeneic WT mice, suggesting that the presence of native IL-10 causes a drift of macrophage function to a proangiogenic phenotype, in contrast to the effect of senescence on macrophage function (Figure 4). Given that IL-10 is such a critical cytokine in regulating abnormal angiogenesis in the eye, we wanted to test the gene expression of IL-10 in the

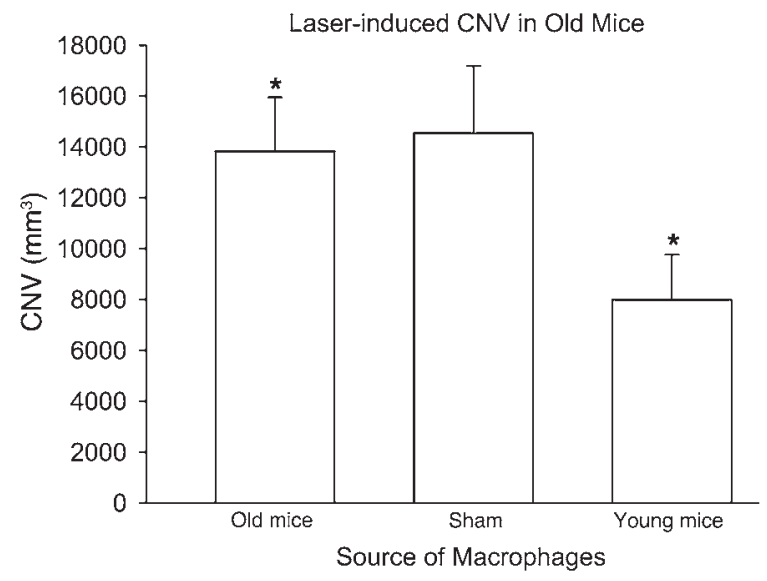



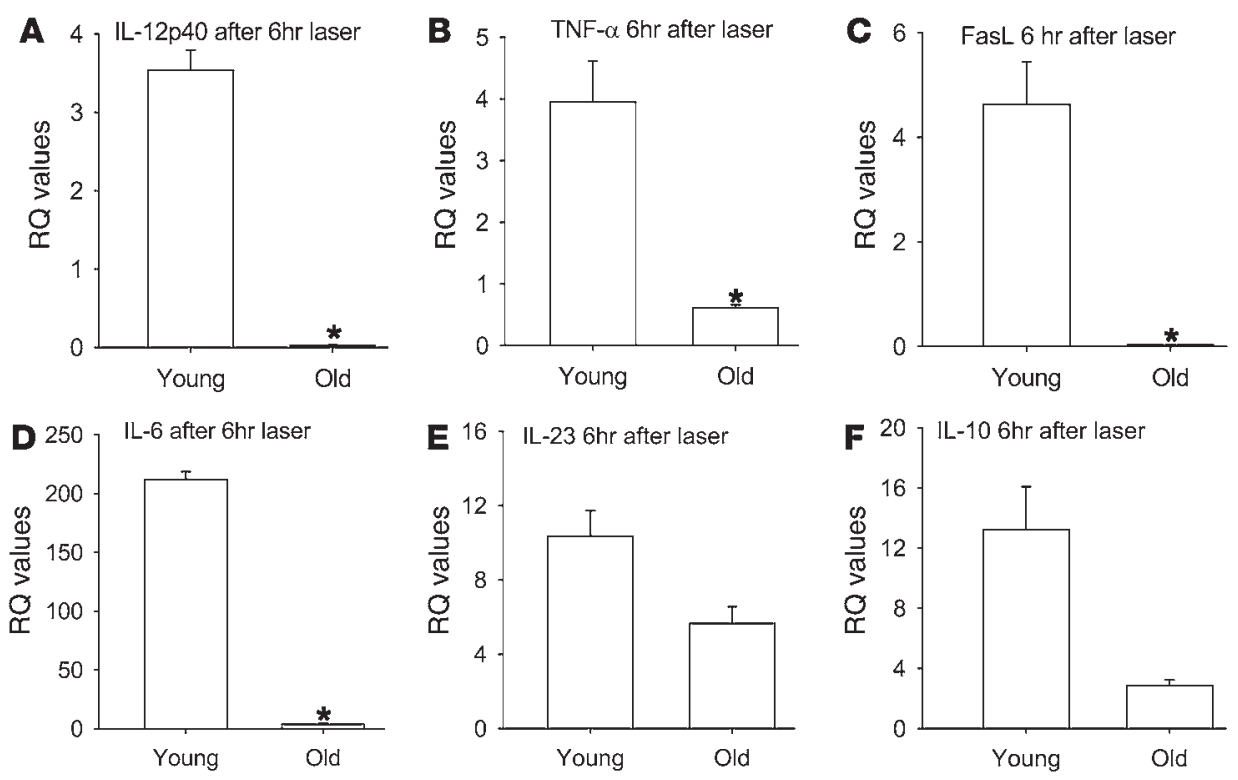

\section{Figure 3}

Quantitative real-time PCR gene expression analysis of macrophage-rich lesions from young and old mouse eyes after laser injury. Matching unlasered tissue was used as the baseline control. Macrophages from old mice demonstrated significant (asterisks) downregulation of $I L-12$ (A, $P=0.0068627), T N F-\alpha$ (B, $P=0.0370)$, and FasL (C, $P=0.0302)$ compared with young mice. IL-6 (D, $P=0.00017)$ and $I L-23(E, P=0.1024)$ were upregulated at levels exponentially higher in young mice than in old mice. (F) IL-10 was upregulated in both young and old mice, although baseline levels of $I L-10(P=0.0701)$ were significantly higher in old mice than young mice. $R Q$, relative quantification. posterior compartment of the eye at baseline in young mice and compare the levels to those of old mice using $I L-10^{-1-}$ eyes as a negative control. As shown in Figure 5, dissected sclerochoroidal tissue from old C57BL/ 6 mice at baseline had exponentially higher levels of IL-10 than did young WT or $I L-10^{-/-}$mice. This result suggests that the micromilieu in old mice is rich in IL-10 and might create an environment that promotes polarization of infiltrating macrophages to a proangiogenic M2 phenotype. In addition, high baseline levels of $I L-10$ in old mice might explain the modest upregulation of $I L-10$ in macrophage-rich lesions of old mice compared with young mice after injury (Figure $3 \mathrm{~F}$ ). We then compared the effect of tissue injury on cytokine gene expression of macrophages within the laser lesions from eyes of $I L-10^{-/-}$ mice with that in C57BL/6 syngeneic WT young mice. Macrophage-enriched CNV lesions from $I L-10^{-/-}$mice demonstrated marked upregulation of $I L-6, T N F-\alpha$, $I L-12$, and Fas $L$ after laser injury in contrast to WT mice (Figure 6, A-D). As expected, $I L-10^{-1-}$ mice had a paucity of IL-10 gene expression, but WT mice had significant upregulation of $I L-10$ after injury (Figure 6E). IL-23 was upregulated in both young and $I L-10^{-/-}$mice (data not shown). This signature defines the $I L-10^{-/-}$macrophage as a TNFhi, IL-6 ${ }^{\text {hi }}$, IL-12 ${ }^{\text {hi }}$, IL-23 ${ }^{\text {hi }}$, FasL ${ }^{\text {hi }}$, antiangiogenic macrophage and is consistent with our results that (a) $I L-10^{-/-}$mice had reduced CNV and (b) $I L-10^{-/-}$mouse-

\section{Figure 4}

Assessment of CNV inhibition following intraocular injection of macrophages $\left(1 \times 10^{5}\right.$ cells/eye). Syngeneic old mice were injected with PBS (sham) or macrophages from young WT or $I L-10^{-/-}$mice on the day of laser, and CNV was measured at day 7 following laser injury. (A) Macrophages isolated from IL-10-/- mice demonstrated significant inhibition of CNV following intraocular injection into old eyes, at levels beyond those seen following intraocular injection of macrophages from young WT mice. * $P<0.008$ vs. sham. (B-D) Representative CNV from mice injected with $I L-10^{-/-}$macrophages (B), young WT macrophages (C), and sham-injected mice (D). derived macrophages inhibited CNV after injection into the eye of a WT mouse. Our results also demonstrated that as mice age, senescent macrophages had elevated IL-10 at baseline and after injury. Old macrophages have a TNF ${ }^{\mathrm{lo}}, \mathrm{IL}_{-} 6^{\text {lo }}, \mathrm{IL}_{-1} 2^{\mathrm{lo}}, \mathrm{IL}-23^{\text {lo }}$, FasL ${ }^{\mathrm{lo}}$, and IL-10 hi phenotype after tissue injury to the eye, a phenotype that impairs their ability to regulate angiogenesis.

Effect of IL-10 and IFN- $\gamma$ on macrophage polarization and angiogenesis. The presence of IL-10, as seen in the tissue micromilieu in old mice, was associated with a proangiogenic phenotype, as seen in Figure 1 and in previous studies (1). In order to directly assess the effect of polarizing cytokines such as IL-10 or IFN- $\gamma$ on macrophages and their regulation of angiogenesis, we isolated $\mathrm{F} 4 / 80^{+}$macrophages from the spleens of WT mice and incubated them with $100 \mathrm{ng} / \mathrm{ml}$
A

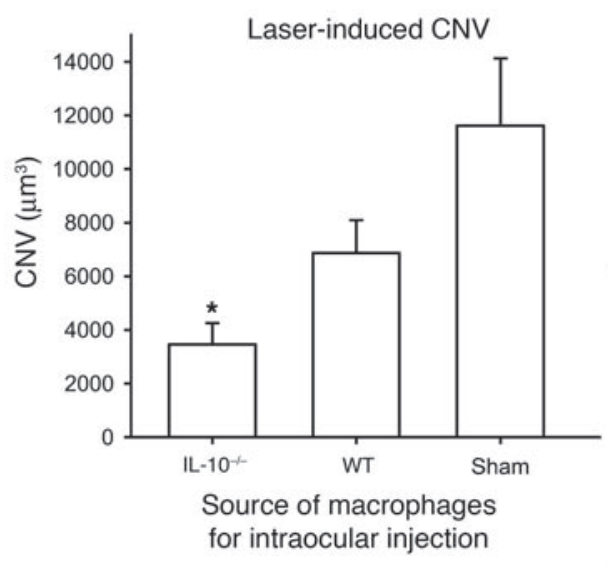

B

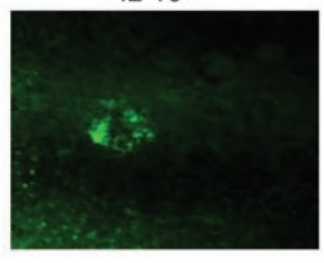

C

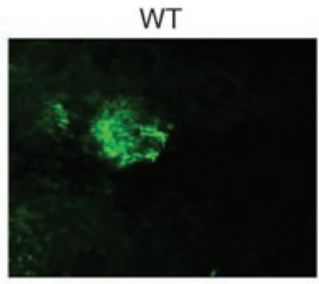

D

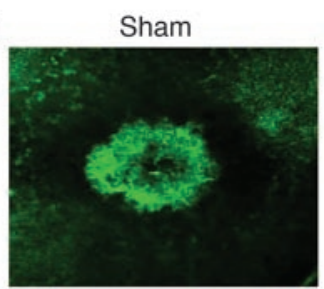




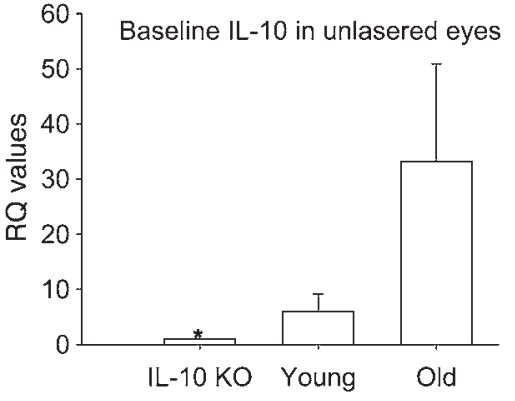

of IL-10 or IFN- $\gamma$ for 24 hours prior to injection. Host WT mice were lasered on day 0 , and their eyes were injected with $1 \times 10^{5}$ $\mathrm{F} 4 / 80^{+}$splenic macrophages. IL-10-treated macrophages failed to inhibit CNV, while IFN- $\gamma$-treated macrophages demonstrated significant inhibition of CNV (Figure 7). These data clearly highlight the important role of IL-10 as a proangiogenic cytokine and demonstrate that in presence of IL-10, macrophages polarize to an M2 phenotype and fail to demonstrate antiangiogenic properties.

Aging impairs the ability of macrophages to regulate vascular endothelial cell proliferation. The results thus far suggest that as mice age, their macrophages lose antiangiogenic function and that IL-10 is a key proangiogenic regulator of macrophage fate and function. We have previously demonstrated that macrophages can directly inhibit vascular endothelial cell proliferation and angiogenesis in vivo (1). We wanted to test directly whether senescence-associated alterations in macrophage gene expression impair the ability of macrophages to inhibit the growth and proliferation of vascular endothelial cells. Thioglycollate-induced peritoneal macrophages (effectors) were harvested from WT young, $I L-10^{-/}$, gld, or old mice and incubated with human dermal microvascular endothelial cells (HMVECs; targets) at a 25:1 ratio in order to assess the effect of macrophages on proliferation of HMVECs. As shown in Figure 8, while macrophages

\section{Figure 5}

Relative gene expression of IL-10 at baseline in dissected sclerochoroidal tissue from $/ L-10^{-/-}$mice, young WT mice, and old WT mice. IL-10 gene expression was performed using real-time PCR; data are represented using the $I L-10^{-/-}$eye as the baseline. ${ }^{\star} P<0.001$ vs. old.

from young and $I L-10^{-/-}$mice significantly inhibited the proliferation of HMVECs, macrophages from old and gld mice failed to do so. The effect was contact dependent: macrophages from gld or old mice placed in transwells above the target HMVECs did not inhibit or stimulate proliferation when compared with macrophages from young or $I L-10^{-/-}$mice placed in transwells (data not shown). This confirms that the loss of antiangiogenic function of senescent macrophages is likely caused by downregulation of FasL and suggests that old macrophages might actually promote abnormal angiogenesis as seen in diseases of aging such as AMD, atheromatous heart disease, and certain cancers.

\section{Discussion}

$\mathrm{CNV}$ in AMD is responsible for more blindness in people over 50 years of age than any other ocular disease $(7,8)$. Our present findings demonstrated that components of innate immunity influenced angiogenesis and that subsets of macrophages exhibited diverse effects on aberrant angiogenesis. Senescence has been shown to influence macrophage function, activation, and polarization (15). In the laser-induced tissue injury model, old mice developed significantly higher volumes of CNV compared with young mice emulating the human disease. Older eyes also had significantly elevated levels of IL-10 at baseline compared with eyes from young mice. Higher levels of IL-10 create a potentially proangiogenic environment in the senescent mouse eye (1). We hypothesized that variations in IL-10 levels in the local micromilieu lead to altered polarization of aged macrophages and macrophages from $I L-10^{-/-}$mice. Quantitative PCR analysis of ocular

\section{Figure 6}

Quantitative real-time PCR gene expression analysis of macrophage-rich lesions from young WT and $I L-10^{-/-}$mouse eyes after laser injury. Matching unlasered tissue was used as the baseline control. (A-D) Macrophages from $/ L-10^{-/-}$ mice demonstrated upregulation of $I L-6(\mathbf{A}, P=0.0558), T N F-\alpha$ (B, $P=0.3579), I L-12(C, P=0.1824)$, and FasL (D, $P=0.4569$ ) compared with young mice. (E) IL-10 was upregulated in young mice and deficient in $I L-10^{-/-}$mice. ${ }^{*} P=0.0513$.
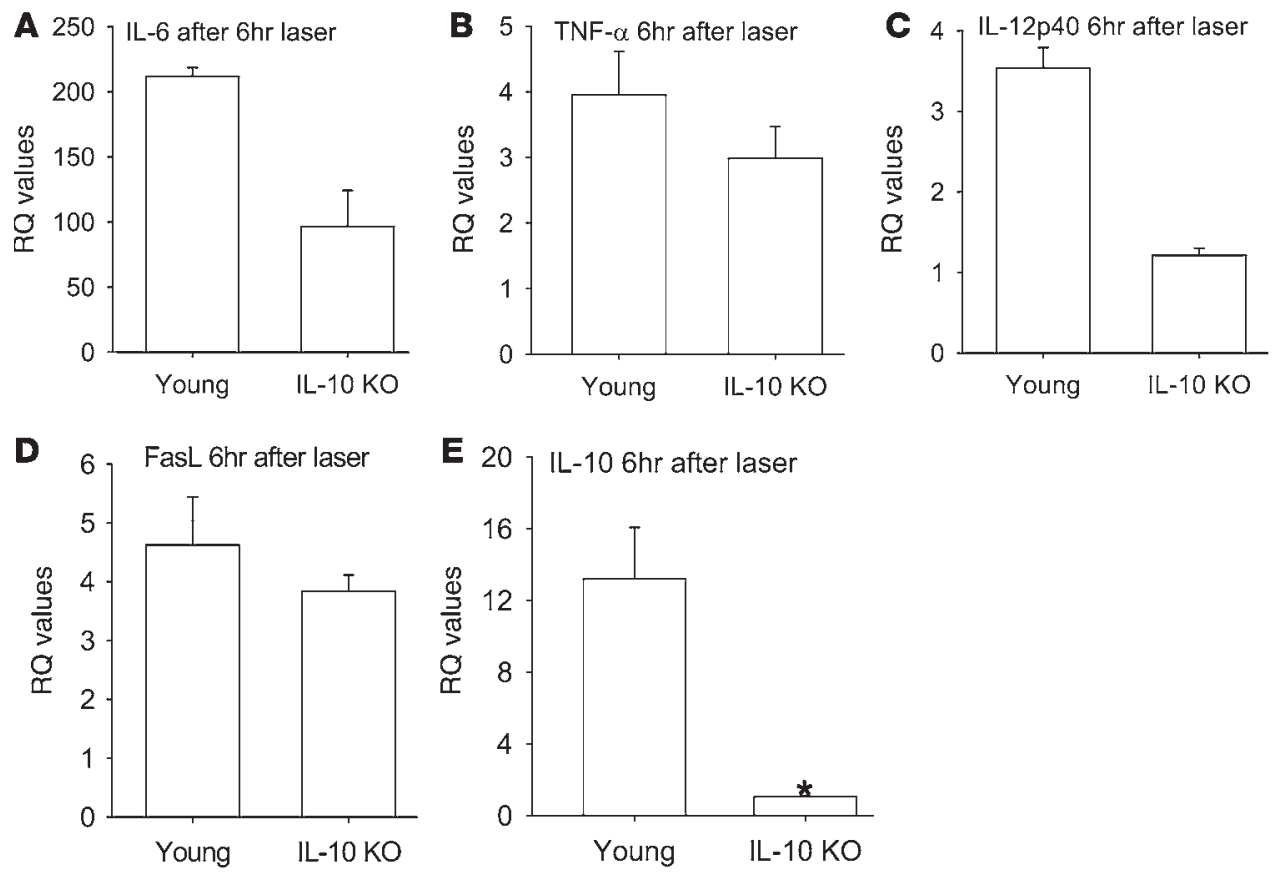


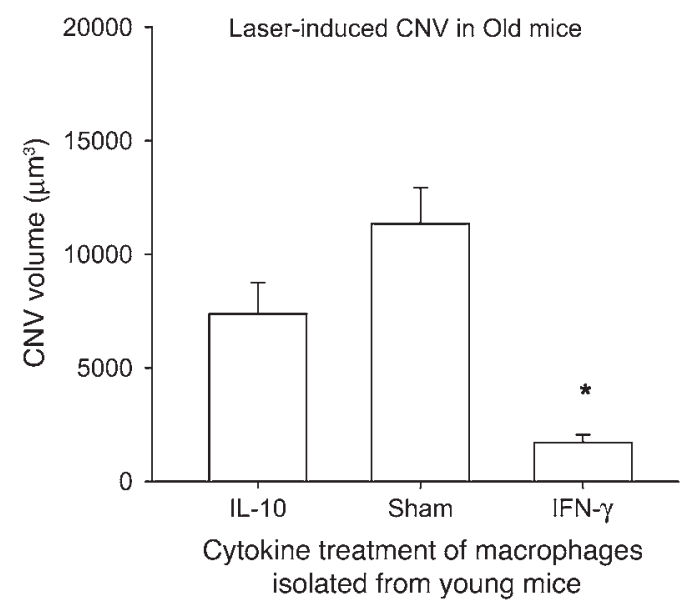

macrophage-rich injured tissue from old mice showed significant upregulation of $I L-10$ after tissue injury, with associated downregulation of Fas $L, I L-12, T N F-\alpha$, and $I F N-\gamma$, a phenotype similar to the more traditionally defined M2 macrophages and one that leads to an impaired ability of senescent macrophages to regulate abnormal angiogenesis. We have previously demonstrated that Fas $L$ on macrophages is critical to the cells' antiangiogenic function (1). In the present study, we demonstrated that reduced expression of FasL on senescent macrophages was associated with an M2 phenotype of these cells and correlated with impaired antiangiogenic function of these cells. In addition, $\mathrm{F} 4 / 80^{+}$macrophages from old mice failed to inhibit CNV upon injection in to the eye and failed to inhibit the proliferation of vascular endothelial cells, consistent with the proangiogenic phenotype of these macrophages.

The results presented here are important to our understanding of macrophages as regulators of angiogenesis. We demonstrated that IL-10 was a key regulator of this process and that a lack of IL-10 in their microenvironment made macrophages antiangiogenic. On the contrary, as macrophages aged, senescence-induced changes in these cells increased baseline levels of $I L-10$ expression. Old macrophages also demonstrate reduced expression of surface Fas $L$ as well as antiangiogenic cytokines such as $I L-12$ and TNF- $\alpha$. This cytokine profile of old macrophages rendered these mice more susceptible to injury-associated angiogenesis. Macrophages from old mice also demonstrated a blunted ability to upregulate IL-6 compared with cells from young mice or IL-10-/ mice. TNF- $\alpha$, IFN- $\gamma$, FasL, IL-12, and IL- 6 are associated with the antiangiogenic macrophage $(1,13)$. A lack of antiangiogenic surveillance function of macrophages may explain why people over 50 years of age are highly susceptible to diseases with dysregulated angiogenesis such as AMD and certain cancers. These results also explain previous studies that have demonstrated that macrophages can be pro- or

\section{Figure 8}

Effect of senescence on macrophage ability to regulate proliferation of vascular endothelial cells. HMVECs were cultured alone, unlabeled $[H(U N L)]$ or labeled $[H(L)]$, or were cultured with macrophages from young WT [M/H(25:1)B6], old WT [M/H(25:1)Old], g/d [M/H(25:1)G/d], and $I L-10^{-/-}\left[\mathrm{M} / \mathrm{H}(25: 1) / L-10^{-/-}\right]$mice for 12 hours prior to measuring the proliferation of HMVEC with $\left[{ }^{3} \mathrm{H}\right]$ thymidine $(\mathrm{TdR})$. Young WT and $I L-10^{-/-}$macrophages inhibited the proliferation of HMVECs, while old and gld macrophages failed to do so. ${ }^{*} P=0.009$; ${ }^{* *} P<0.00086$.

\section{Figure 7}

Ability of macrophages to inhibit CNV after treatment with polarizing cytokines. $\mathrm{F} 4 / 80^{+}$macrophages were isolated from the spleens of C57BL/6 young WT mice, treated with $100 \mathrm{ng} / \mathrm{ml} \mathrm{IL-10} \mathrm{or} \mathrm{IFN-} \gamma$, and then injected in the eyes of host WT mice on the day of laser $\left(1 \times 10^{5}\right.$ cells/eye). Macrophages treated with IFN- $\gamma$ significantly inhibited CNV, while macrophages treated with IL-10 failed to do so. ${ }^{*} P<0.000003$ vs. sham.

antiangiogenic (11-13). Polarization of macrophages determines their ability to regulate angiogenesis. It might be possible in the future to design immunotherapies that could potentially exploit the ability to convert macrophages to an antiangiogenic phenotype by disrupting IL-10 function in order to limit complications of AMD such as visual impairment and blindness.

\section{Methods}

Laser-induced model of CNV. Rupture of Bruch's membrane with laser was used to initiate CNV in 5- to 7-week-old mice as described previously (1). Briefly, mice were anesthetized using intraperitoneal ketamine hydrochloride $(86.9 \mathrm{mg} / \mathrm{kg})$ and xylazine $(13.4 \mathrm{mg} / \mathrm{kg})$, and their pupils were dilated with $1 \%$ tropicamide. Using krypton red laser, 4 laser burns were placed around the optic nerve ( 0.05 seconds, $50 \mu \mathrm{m}, 150 \mathrm{~mW})$. Seven days after laser, the mice were anesthetized as described above and perfused intraventricularly with fluorescein-labeled dextran. The mice were euthanized with $\mathrm{CO}_{2}$ inhalation, and their eyes were harvested for tissue processing. A dissecting microscope was used to remove the cornea and lens and to gently separate the retina from the underlying choroid and sclera. Microscissors were used to make 4 radial incisions in the sclerochoroidal "eyecup" in order to prepare choroidal flat mounts on glass slides. The tissues were incubated in $4 \%$ paraformaldehyde for 45 minutes and washed 3 times with $3 \%$ bovine serum albumin. A drop of fixative (Prolong) and a glass coverslip were placed on each slide. The choroidal flat mounts were analyzed for presence of CNV by confocal microscopy. The extent of choroidal neovascularization was quantified by Metamorph Imaging software.

Animal research. Mice were purchased from The Jackson Laboratory (C57BL/6 WT, $I L-10^{-/}$) or from the National Institute for Aging, NIH (C57BL/ 6 old mice, $>18$ months of age). All animal experiments were approved by the Animal Studies Committee at Washington University in St. Louis School of Medicine. All experiments contained at least 3 mice per group and were repeated a minimum of 2 times.

Positive selection of macrophages and intraocular injection. Positive selection of $\mathrm{F} 4 / 80^{+}$macrophages was performed as described previously (1). Briefly,

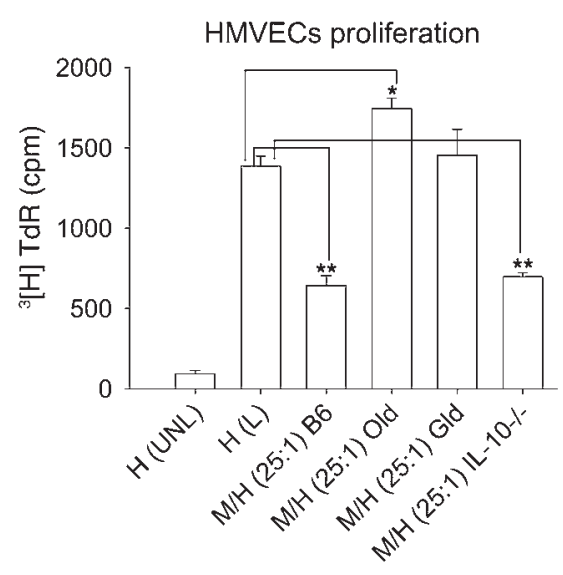


$\mathrm{F} 4 / 80^{+}$macrophages were purified from dissociated mouse spleen cells by positive selection using magnetic separation (Stemcell Technologies Inc.). Cell purity was greater than $90 \%$ by flow cytometry. Cells were injected into the vitreous cavities of mouse eyes using a 33-gauge Hamilton needle on the same day as laser treatment. We used $1 \times 10^{5}$ macrophages for intravitreal injections based on previously published dose-response experiments (1). Control mice were injected with PBS.

Isolation of ocular macrophages. Sclerochoroidal eyecups were made from eyes with or without prior laser injury as described above. We have previously demonstrated that macrophages migrate into the laser-induced tissue injury lesions in the choroid (1). In eyes subjected to laser injury, careful attention was paid to dissecting out the laser lesions containing the infiltrating macrophages for gene expression analysis. The presence of macrophages within the laser lesions at 6 hours was confirmed by immunostaining with F4/80-APC antibody (data not shown). Unlasered matching sclerochoroidal tissue from syngeneic mice was obtained as a control for comparison and quantification.

Real-time PCR and gene expression analysis. Total RNA was prepared from harvested ocular tissue 6 hours after laser injury using the RNeasy mini kit (Qiagen). Unlasered ocular tissue was harvested as control. RNA was purified by a gDNA eliminator spin column in order to minimize the amount of gDNA cross-contamination. cDNA was prepared using the High Capacity cDNA Archive Kit (Applied Biosystems). Relative levels of target gene expression were measured on the 7500 Real-time RT-PCR system (Applied Biosystems). For fluorescence measurement and assay preparation, FAM490-based Taqman Gene Expression Assay Mix (Applied Biosystems) specific for each gene of interest and Taqman Universal Master Mix (Applied Biosystems) were used. Relative Quantification PCR analysis was performed using the ABI 7500 SDS Software. The threshold cycle $(\mathrm{Ct})$ values were measured for our endogenous control ( $\beta$-actin or 18 s rRNA) and the genes of interest at the logarithmic phase of amplification. The $\Delta \mathrm{Ct}$ values for the calibrator (negative baseline control) were calculated as the difference between the $\mathrm{Ct}$ value of the endogenous control and the target gene of interest. This $\Delta \mathrm{Ct}$ value for the calibrator was then compared with the $\Delta \mathrm{Ct}$ value of each of our unknown cDNA samples, and the difference of these 2 values was taken to achieve the $\Delta \Delta \mathrm{Ct}$ value for each gene. The relative fold expression difference was calculated as $2^{-\Delta \Delta \mathrm{Ct}}$ and graphed.

Taqman gene expression assay mixes. Primer and probe sets were as follows: ActB, Mm00607939_s1; 18s rRNA, Hs99999901_s1; FasL, Mm00438864_ m1; TNF- $\alpha, M m 00443258 \_m 1 ; I L-6, M m 00446190 \_m 1 ; I L-10$, Mm00439616_m1; IL-23, Mm00518984_m1; IL-12, Mm01288989_m1. Data are represented as gene expression increase or decrease in macrophage-rich eye lesions from old, young, or $I L-10^{-/-}$mice after injury compared with uninjured tissue within the same group.

Vascular endothelial cell proliferation assay. Young WT C57BL/6 (6-8 weeks of age), syngeneic $I L-10^{--}$, syngeneic gld (FasL-deficient), and syngeneic old mice were injected intraperitoneally with $3 \%$ thioglycollate for 5 days. Macrophages were harvested with sterile PBS from the peritonea of these mice and cultured in RPMI-1640 plus 10\% FCS overnight. Macrophages were then washed with RPMI-1640 to remove nonadherent cells and stimulated with LPS $(100 \mathrm{ng} / \mathrm{ml})$ for 12 hours. Subsequently, HMVECs $\left(5 \times 10^{4}\right.$ cells; Lonza) in log phase were put in EGM2V (Lonza) in 96-well round-bottomed plate for adherence. After stimulation, macrophages were washed to remove LPS and cocultured with HMVECs for another 12 hours. Macrophages were then flushed by repeated and gentle pipetting; HMVECs were incubated with $40 \mu \mathrm{Ci} / \mathrm{ml}\left[{ }^{3} \mathrm{H}\right]$ thymidine (TRA61; GE Health Care) for 12 hours. Plate was harvested and read using a Topcount harvester and micro plate reader (Packard).

Statistics. One-tailed Student's $t$ test was used for statistical analysis. $P$ less than 0.05 was considered statistically significant.

\section{Acknowledgments}

This work was supported by NIH grants K08EY016139 and Diabetes Research and Training Center 5 P60 DK20579, a Carl Marshall Reeves and Mildred Almen Reeves Foundation Inc. Award, a Research to Prevent Blindness Career Development Award, and an American Federation for Aging Research Grant (to R.S. Apte); by NIH grants EY12826 and EY06765 (to T.A. Ferguson); and by Department of Ophthalmology and Visual Sciences core grant EY02687.

Received for publication April 18, 2007, and accepted in revised form August 15, 2007.

Address correspondence to: Rajendra S. Apte, Department of Ophthalmology and Visual Sciences, Washington University School of Medicine, 660 South Euclid Avenue, Box 8096, St. Louis, Missouri 63110, USA. Phone: (314) 747-5262; Fax: (314) 362-6793; E-mail: apte@vision.wustl.edu.
1. Apte, R.S., Richter, J., Herndon, J., and Ferguson, T.A. 2006. Macrophages inhibit neovascularization in a murine model of Age-related macular degeneration. PLoS Med 3:e310-e320.

2. Nakao, S., et al. 2005. Infiltration of COX-2expressing macrophages is a prerequisite for IL-1 $\beta$ induced neovascularization and tumor growth. J. Clin. Invest. 115:2979-2991. doi:10.1172/JCI23298.

3. Taylor, P.R., et al. 2005. Macrophage receptors and immune recognition. Annu. Rev. Immunol. 23:901-944.

4. Hansson, G.K. 2005. Inflammation, atherosclerosis, and coronary artery disease. N. Engl. J. Med. 352:1685-1695.

5. Lobov, I.B., et al. 2005. WNT7b mediates macrophage-induced programmed cell death in patterning of the vasculature. Nature. 437:417-421.
6. Ambati, J., et al. 2003. An animal model of agerelated macular degeneration in senescent $\mathrm{Ccl}-2$ - or Ccr-2-deficient mice. Nat. Med. 9:1390-1397.

7. van Leeuwen, R., Klaver, C.C., Vingerling, J.R., Hofman, A., and de Jong, P.T. 2003. Epidemiology of age-related maculopathy: a review. Eur. J. Epidemiol. 18:845-854.

8. Klein, R., Peto, T., Bird, A., and Vannewkirk, M.R. 2004. The epidemiology of age-related macular degeneration. Am. J. Ophthalmol. 137:486-495.

9. Espinosa-Heidmann, D.G., et al. 2003. Macrophage depletion diminishes lesion size and severity in experimental choroidal neovascularization. Invest. Ophthalmol. Vis. Sci. 44:3586-3592.

10. Lamagna, C., Aurrand-Lions, M., and Imhof, B.A. 2006. Dual role of macrophages in tumor growth and angiogenesis. J. Leukoc. Biol. 80:705-713.
11. Mantovani, A., Sica, A., and Locati, M. 2005. Macrophage polarization comes of age. Immunity. 23:344-346.

12. Sica, A., Schioppa, T., Mantovani, A., and Allavena, P. 2006. Tumour-associated macrophages are a distinct M2 polarised population promoting tumour progression: potential targets of anti-cancer therapy. Eur. J. Cancer. 42:717-727.

13. Mosser, D.M. 2003. The many faces of macrophage activation. J. Leukoc. Biol. 73:209-212.

14. Espinosa-Heidmann, D.G., et al. 2002. Age as an independent risk factor for severity of experimental choroidal neovascularization. Invest. Ophthalmol. Vis. Sci. 43:1567-1573.

15. Gomez, C.R., Boehmer, E.D., and Kovacs, E.J. 2005. The aging innate immune system. Curr. Opin. Immunol. 17:457-462. 